\title{
Simulating coalitionally stable burden sharing agreements for the climate change problem*
}

\author{
Johan Eyckmans ${ }^{\dagger}$ \\ Katholieke Universiteit Leuven, Centrum voor Econmische Studiën \\ Henry Tulkens \\ Université Catholique de Louvain, Center for Operations Research and Econometrics CORE
}

first version: January 1999

this version: June 2003

forthcoming in Resource and Energy Economics

\begin{abstract}
The CLIMNEG World Simulation (CWS) model is introduced here for simulating cooperative game theoretic aspects of global climate negotiations. The CWS model is derived from the seminal RICE model by Nordhaus and Yang (1996). We first state the necessary conditions that determine Pareto efficient investment and emission abatement paths under alternative regimes of cooperation between the regions. We then show with a numerical version of the CWS model that the transfer scheme advocated by Germain, Toint and Tulkens (1997) induces an allocation in the ("gamma") core of the world carbon emission abatement cooperative game.
\end{abstract}

Keywords: environmental economics, climate change, burden sharing, simulations, core of cooperative games

JEL codes: C71, C73, D9, D62, F42, Q2

${ }^{*}$ This research is part of the CLIMNEG project, funded by the Belgian Federal Office for Scientific, Technical and Cultural Affairs (OSTC), contracts CG/DD/241 and CG/DD/243. The authors wish to express their thanks to Professor William Nordhaus for making his program code available to them, to Marc Germain for careful reading as well as to their colleagues from UcL, KULeuven and Bureau Fédéral du Plan/Federaal Planbureau in the CLIMNEG team for stimulating discussions in numerous working sessions. Useful comments and suggestions by two anonymous referees, by Carlo Carraro, by Juan C. Cìscar and by Jacques Drèze are gratefully acknowledged. All remaining errors remain, of course, ours.

${ }^{\dagger}$ corresponding author: Johan Eyckmans, Katholieke Universiteit Leuven, Centrum voor Economische Studiën CES, Naamsestraat 69, B-3000 Leuven, Belgium, tel + 32163268 44, fax + 3216326910 , e-mail johan.eyckmans@econ.kuleuven.ac.be 


\section{Introduction and Summary}

International environmental agreements involving substantial emission reduction efforts are unlikely to be reached without provisions for international transfers. The reason is that, although there generally is a substantial surplus to be gained from cooperation, there are most often countries for which the abatement effort required by the World optimum is so large that they end up worse off under this World optimum compared to the noncooperative laissez-faire situation. The World optimum thereby appears to be unstable. That international transfers can compensate for such undesirable outcome was argued quite long ago (e.g., Tulkens, 1979) and taken up again more recently, albeit with some scepticism, by Carraro and Siniscalco (1993) or Barrett (1994).

This paper investigates how such international transfers might look like in the case of the global climate change problem. In particular we employ a variant of the transfer scheme initially proposed by Chander and Tulkens (1995 and 1997)(CT hereafter) in a static context and extended by Germain, Toint and Tulkens (1997)(GTT hereafter) to a dynamic context. Essentially the scheme consists in redistributing the surplus of cooperation over non-cooperation in proportion ${ }^{1}$ to the (marginal) climate change damage costs that countries experience. In these papers, it is shown that the proportional transfer scheme results in an allocation in the core of a cooperative emission abatement game. This core property is a necessary (but not sufficient) condition for full, voluntary cooperation among the countries involved as explained in Tulkens (1998). If it is not satisfied, coalitions of countries can obtain a better outcome by coordinating their emission strategies among themselves and such coalitions have no incentive to join a worldwide environmental treaty.

The core results just mentioned have been obtained only under a linearity assumption of the damage cost functions, which is hard to maintain for the climate change problem. Do these results still hold in the nonlinear case? Given the absence of an analytical answer, we may turn to an empirical model and test the core property of the transfer scheme numerically.

For this purpose we introduce in Section 2 the CLIMNEG World Simulation (CWS) model which we derived from the seminal multi-region economy-climate RICE model of Nordhaus and Yang (1996). Like RICE, the CWS model is an optimal growth model for the World economy, coupled with a basic representation of the carbon cycle and climate system. The main differences between RICE and CWS are that we work with a model in which utilities are linear in consumption and that we do not consider international trade flows. Both of these modifications are motivated below; they are made essentially to focus on the game theoretic aspects of the cooperation problem.

\footnotetext{
${ }^{1}$ For international environmental agreements, proportionality w.r.t. damages has been advocated not only for its strategic stability properties, but also for incentive compatibility reasons, see for instance Eyckmans (1997).
} 
In Section 3 we describe various scenarios w.r.t. cooperation in the CWS model. First, absence of cooperation is modelled as an open-loop Nash equilibrium of carbon emissions. Also a Ramsey-Keynes rule is formulated, determining an inter-temporal allocation of investment and consumption that disregards spillovers to neighboring countries. Next, Pareto efficient allocations are characterized. In such allocations, the rule determining emission abatement efforts is shown to be a dynamic version of the Samuelson (1954) rule for the optimal provision of public goods. Parallel to that, capital accumulation is determined by a generalization of the Keynes-Ramsey rule that internalizes the carbon emission externalities.

Contrasting Nash equilibria and Pareto efficient allocations of abatement effort is not new to the literature: the point has been made before by, among others, Mäler (1989), Hoel (1992), Van der Ploeg and De Zeeuw (1992) and Kaitala, Pohjola and Tahoven (1992). In this paper, however, we go one step further by characterizing intermediate scenarios of incomplete cooperation by subgroups (coalitions) of countries. To that effect, we define partial agreement Nash equilibria with respect to a coalition which is the counterpart for dynamic models of a concept introduced in CT. Optimality rules driving investment and emission abatement decisions at such equilibria turn out to be a combination of the optimality rules for the Pareto efficient allocations and the open-loop Nash equilibrium.

In Section 4 the partial agreement Nash equilibrium w.r.t. a coalition concept is used to define the core of a carbon emission abatement cooperative game. Core allocations satisfy both individual and coalitional participation constraints, i.e. these allocations are such that no individual country, nor any coalition of countries, can gain by returning to its partial agreement Nash equilibrium.

In section 5 simulations with the numerical CWS model are reported. We first compute three reference scenarios (business-as-usual, Nash equilibrium and Pareto efficient allocation without transfers) and we compare them in terms of carbon emissions, carbon concentrations, temperature change, emission abatement effort and consumption levels over the period of analysis. The value of cooperation over non-cooperation is estimated on that basis.

We next check for the core property in the numerical CWS model. We therefore compute all possible partial agreement Nash equilibria w.r.t. any coalition and compare each coalition's payoff to its joint allocation of consumption under the Pareto efficient solution without transfers. We observe in the simulations that the core property is violated, both for China as an individual region, and for several intermediate coalitions containing both China and Rest Of the World. We then consider whether the participation problem raised thereby can be overcome using the GTT international transfer scheme appropriately modified to allow for nonlinear damage cost functions. It turns out that all coalitions are better off under the transfer scheme than under their respective partial agreement Nash equilibrium. Hence, the Pareto efficient scenario with transfers belongs to the core of the game; in this sense, it can be sustained as a voluntary agreement. 
We also exploit our study of the 64 conceivable coalitions to evaluate some post-Kyoto partial cooperation policies: cooperation within Annex B, the US non-cooperation, and cooperation within Annex B without the US. Finally, we point to the necessity of extending this analysis to closed-loop Nash equilibria so as to allow for transfers defined at every point in time rather than as single number discounted aggregates. Our conclusion in Section 6 emphasizes the specific merits of the cooperative game theoretic approach in the framework of international environmental agreements.

\section{CWS, an integrated climate-economy World model}

\section{$2.1 \quad$ Statement of the model}

In the CWS model, each national economy is represented by a discrete time optimal growth model with a long but finite horizon. Growth is driven by exogenous population growth and technological change as well as by endogenous capital accumulation. Letting $N$ denote the set of countries/regions ${ }^{2}$ indexed $i=1,2, \ldots, n$, the following equations describe the economy of region $i$ at time $t$ :

$$
\begin{aligned}
Y_{i, t} & =Z_{i, t}+I_{i, t}+C_{i}\left(\mu_{i, t}\right)+D_{i}\left(\Delta T_{t}\right) \\
Y_{i, t} & =A_{i, t} F_{i}\left(K_{i, t}\right) \\
K_{i, t+1} & =\left[1-\delta_{K}\right] K_{i, t}+I_{i, t} ; \quad K_{i, 0} \text { given }
\end{aligned}
$$

Equation (1) defines the claims of consumption $Z_{i, t}$, investment $I_{i, t}$, cost of abatement $C_{i}\left(\mu_{i, t}\right)$ and climate change damage $D_{i}\left(\Delta T_{t}\right)$ upon production $Y_{i, t}$. The costs of abatement and of climate change damages are both assumed to be strictly increasing and strictly convex in abatement $\mu_{i, t}$ and temperature change $\Delta T_{t}$ respectively. (2) defines production as a strictly increasing and strictly concave function of capital input $K_{i, t}$. $A_{i, t}$ measures overall productivity. It is assumed that productivity increases exogenously as time goes by and technological progress is Hicks neutral. Population, identified with labor supply, is assumed to be an exogenous input in production and is subsumed in the productivity factor $A_{i, t}$. Finally, expression (3) is a capital accumulation equation where $\delta_{K}$ stands for the rate of capital depreciation.

This economic model is coupled with a simple climatic model of global mean temperature change. The carbon emissions, the carbon cycle and their effect on climate are respectively

\footnotetext{
${ }^{2}$ In the sequel we will indifferently speak of regions or countries, even if a region contains only one country.
} 
modelled by the following equations:

$$
\begin{aligned}
E_{i, t} & =\sigma_{i, t}\left[1-\mu_{i, t}\right] Y_{i, t} \\
M_{t+1} & =\left[1-\delta_{M}\right] M_{t}+\beta \sum_{i \in N} E_{i, t} \quad ; \quad M_{0} \text { given } \\
\Delta T_{t} & =G\left(M_{t}\right)
\end{aligned}
$$

According to expression (4), carbon emissions $E_{i, t}$ are proportional to production. The emissions to output ratio $\sigma_{i, t}$ declines exogenously over time due to an assumed autonomous energy efficiency increase. Emissions can be reduced at a rate $\mu_{i, t} \in[0,1]$ in every period though this is costly according to equation (1). Equation (5) describes the accumulation of carbon in the atmosphere. This process is modelled similarly to a standard capital accumulation process where $\delta_{M}$ denotes the natural decay rate of atmospheric carbon concentrations and $\beta$ is the airborne fraction of carbon emissions.

Expression (6) translates atmospheric carbon concentration levels into global mean temperature change ${ }^{3}$. We assume that $G$ is a continuously differentiable and increasing function. For the purpose of this section, there is no need to make the function $G$ more explicit. In the numerical simulation of CWS, we will adopt exactly the same formulation as in RICE for the carbon cycle and temperature change equations; this formulation satisfies the general properties we assume for $G$ presently.

Finally, the welfare $W_{i}$ of each country $i$ is measured by its aggregate lifetime discounted consumption:

$$
W_{i}=\sum_{t=0}^{T} \frac{Z_{i, t}}{\left[1+\rho_{i}\right]^{t}}+w_{i}\left(K_{i, T+1}\right)
$$

where $\rho_{i}$ stands for the discount rate of region $i$ and the strictly increasing and strictly concave function $w_{i}$ stands for the scrap value of the terminal capital stock $K_{i, T+1}$.

\subsection{Differences with the RICE model}

Conceptually, the model outlined above is very similar to the RICE model by Nordhaus and Yang (1996). In this section we clarify and motivate the differences that our formulation introduces. First, we do not allow for international trade. Trade complicates the

\footnotetext{
${ }^{3}$ This formulation is an extreme simplification of the physical processes behind climate change. In Eyckmans and Bertrand (2000) we provide a more realistic model of the carbon cycle and climate change process which takes into account regional differences in temperature change and cooling from sulphate aerosols.
} 
analysis considerably because it creates, besides the climate change externality, additional interdependencies between the regions that we want to avoid in order to better concentrate on the cooperation issues raised by the environmental externality. In addition, we feel that the way Nordhaus and Yang (1996) introduce trade in RICE is not fully satisfactory. In their Negishi solution, what they call exports are in fact some kind of (restricted) normative transfers among the regions whose justification is not clear. Finally, since the magnitude of actual net exports (exports minus imports) is relatively small, we feel that not much is lost from leaving out international trade.

Secondly, we use an additive instead of a multiplicative formulation of the effect of emission abatement costs and climate change damages on consumption possibilities. Translated into our notation, Nordhaus and Yang's formulation of the budget equation (1) is given by:

$$
\Omega_{i, t} Y_{i, t} \equiv \frac{1-C_{i}\left(\mu_{i, t}\right) / Y_{i, t}}{1+D_{i}\left(\Delta T_{t}\right) / Y_{i, t}} Y_{i, t}=Z_{i, t}+I_{i, t}
$$

Conceptually, the two formulations reflect the same fact that costs of emission abatement and of damages from climate change reduce the amount of production that can be devoted to consumption or investment. The difference lies in the fact that Nordhaus and Yang (1996) allow for cross effects between emission abatement costs and climate change damages: this type of cross effects are excluded by our formulation.

Thirdly, in contrast to Nordhaus and Yang (1996), we assume that utilities are linear in consumption. We make this simplification in order to represent the global carbon emission game as a transferable utility (TU) game. For our purpose of game theoretic stability analysis by means of numerical simulations, a TU framework is better suited than a nonTU game. In particular, for the cooperative solution concept of the core in a non-TU context, one cannot use the additive form of the worth of a coalition on which our present computations rest.

Fourthly, the CWS model allows for different regional discount rates. We feel that the huge differences between World regions in terms of economic development and openness to financial markets do not justify that a uniform discount rate be applied in all regions, as is the case in RICE. In our simulations, we have chosen systematically higher discount rates for developing regions than for industrialized countries. 


\section{Alternative scenarios as to cooperation in the CWS model}

\subsection{Nash equilibrium}

We first describe what would happen if the regions do not sign a voluntary international environmental agreement. We characterize such a situation by means of the concept of open-loop Nash equilibrium. An open-loop Nash equilibrium (Nash equilibrium hereafter) is a family of strategies, one for each player, that maximize every region $i$ 's welfare, given the strategies of all other players $j \neq i$. In such an equilibrium, no individual region has an incentive to deviate as long as the other regions stick to their equilibrium strategies.

In our CWS model, a Nash equilibrium is obtained by maximizing every region's welfare (7) with respect to its control variables $I_{i, t}, Z_{i, t}$ and $\mu_{i, t}$ while respecting its individual resource and capital constraints and the climate modules for given emissions $\bar{E}_{i, t}$ of all other regions $j \neq i$ and $\forall t$. Formally, for region $i$ :

$$
\max _{\left\{Z_{i, t}, I_{i, t}, \mu_{i, t}\right\}_{t=0, \ldots, T}} W_{i}=\sum_{t=0}^{T} \frac{Z_{i, t}}{\left[1+\rho_{i}\right]^{t}}+w_{i}\left(K_{i, T+1}\right)
$$

subject to (for all $0 \leq t \leq T$ ):

$$
\begin{aligned}
A_{i, t} F_{i}\left(K_{i, t}\right) & \geq Z_{i, t}+I_{i, t}+C_{i}\left(\mu_{i, t}\right)+D_{i}\left(G\left(M_{t}\right)\right) & \forall i, t & {\left[\zeta_{i, t}\right] } \\
K_{i, t+1} & =\left[1-\delta_{K}\right] K_{i, t}+I_{i, t} & \forall i, t & {\left[\psi_{i, t}\right] } \\
M_{t+1} & =\left[1-\delta_{M}\right] M_{t}+\beta \sigma_{i, t}\left[1-\mu_{i, t}\right] A_{i, t} F_{i}\left(K_{i, t}\right)+\beta \sum_{j \neq i} \bar{E}_{j, t} & \forall t & {\left[\phi_{i, t}\right] }
\end{aligned}
$$

In addition, initial values of the state variables $K_{i, 0}$ and $M_{0}$ are given, all variables are constrained to be non-negative and $\mu_{i, t} \leq 1$. We associate Lagrange multipliers $\zeta_{i, t}$ to the resource constraint ${ }^{4}, \psi_{i, t}$ to the capital accumulation constraint and $\phi_{i, t}$ to the carbon accumulation process. Differentiating with respect to all variables we get the following firstorder conditions for an interior optimum (the superscript "NE" refers to the equilibrium

\footnotetext{
${ }^{4}$ Since the objective is monotonically increasing in consumption $Z_{i, t}$, the inequality in the resource constraint can be replaced by an equality and a Lagrange multiplier can be used.
} 
values of the variables at the Nash equilibrium):

$$
\begin{aligned}
\zeta_{i, t}^{N E}= & \frac{1}{\left[1+\rho_{i}\right]^{t}}=\psi_{i, t}^{N E} & \forall i, t \\
\zeta_{i, t}^{N E} C_{i}^{\prime}\left(\mu_{i, t}^{N E}\right)= & \beta \sigma_{i, t} A_{i, t} F_{i}\left(K_{i, t}^{N E}\right) \phi_{i, t}^{N E} & \forall i, t \\
\psi_{i, t-1}^{N E}= & \psi_{i, t}^{N E}\left[A_{i, t} F_{i}^{\prime}\left(K_{i, t}^{N E}\right)+\left[1-\delta_{K}\right]\right] & \\
& -\beta \sigma_{i, t}\left[1-\mu_{i, t}^{N E}\right] A_{i, t} F_{i}^{\prime}\left(K_{i, t}^{N E}\right) \phi_{i, t}^{N E} & \forall i, t \\
\psi_{i, T}^{N E}= & w_{i}^{\prime}\left(K_{i, T+1}^{N E}\right) & \forall i \\
\phi_{i, t-1}^{N E}= & G^{\prime}\left(M_{t}^{N E}\right) \zeta_{i, t}^{N E} D_{i}^{\prime}\left(G\left(M_{t}^{N E}\right)\right)+\left[1-\delta_{M}\right] \phi_{i, t}^{N E} & \forall i, t \\
\phi_{i, T}^{N E}= & 0 & \forall i
\end{aligned}
$$

A Nash equilibrium of the CWS model is a solution to this system of first-order conditions, holding simultaneously for all $i \in N$. Conditions (9) say that for every region $i$ and in every period $t$ the shadow cost of capital equals the shadow cost of the resource constraint and that both are equal to the discount factor of region $i$. (10) determines the optimal amount of carbon emissions control for country $i$. The evolution of the capital stock is described by conditions (11) for all but the last period and (12) for the last period. Expression (13) describes the evolution of the Lagrange multiplier associated with the atmospheric carbon concentration equation. In economic terms, this multiplier can be interpreted as the shadow price of carbon concentration. Finally, condition (14) says that last period's shadow price of carbon equals zero.

By using the terminal condition $\phi_{i, T}^{N E}=0$ for solving iteratively the difference equation (13), it appears that the shadow price of carbon for region $i$ in period $t$ is equal to the sum of all its future marginal damages caused by an additional unit of carbon emissions at time $t$ :

$$
\phi_{i, t}^{N E}=\sum_{\tau=t+1}^{T}\left[1-\delta_{M}\right]^{\tau-t-1} G^{\prime}\left(M_{\tau}^{N E}\right) \frac{D_{i}^{\prime}\left(G\left(M_{\tau}^{N E}\right)\right)}{\left[1+\rho_{i}\right]^{\tau}}
$$

Notice that, since (15) holds for each region $i$ separately, the shadow price of atmospheric carbon concentration at a Nash equilibrium only takes into account the climate change damage occurring within a region's territory. Spillover effects to neighboring regions are not taken into account in the region's individual decision process.

Substituting (15) for the shadow price in (10), we derive for each country $i$ a rule determining its optimal amount of carbon emission control at a Nash equilibrium, that reads as 
follows:

$$
\frac{1}{\left[1+\rho_{i}\right]^{t}} \frac{C_{i}^{\prime}\left(\mu_{i, t}^{N E}\right)}{\sigma_{i, t} A_{i, t} F_{i}\left(K_{i, t}^{N E}\right)}=\beta \phi_{i, t}^{N E}=\beta \sum_{\tau=t+1}^{T}\left[1-\delta_{M}\right]^{\tau-t-1} G^{\prime}\left(M_{\tau}^{N E}\right) \frac{D_{i}^{\prime}\left(G\left(M_{\tau}^{N E}\right)\right)}{\left[1+\rho_{i}\right]^{\tau}}
$$

The left hand side (LHS) stands for the discounted marginal cost for region $i$ of reducing its carbon emissions by an additional ton in period $t$. The denominator denotes gross emissions without abatement and is used to convert the units of the marginal abatement costs into US\$ per ton of carbon ${ }^{5}$. (16) is the traditional optimality condition for a noncooperative Nash equilibrium, saying that marginal abatement costs should be equal to individual marginal damage of climate change.

We now turn to the Ramsey-Keynes condition that drives capital accumulation for country $i$. Substituting (9) into (12), the latter condition can be written as follows:

$$
\rho_{i}+\delta_{K}=A_{i, t} F_{i}^{\prime}\left(K_{i, t}^{N E}\right)\left[1-\beta \sigma_{i, t}\left[1-\mu_{i, t}^{N E}\right]\left[1+\rho_{i}\right]^{t} \phi_{i, t}^{N E}\right]
$$

This condition says that along an optimal investment path, region $i$ should be indifferent between consuming an additional $\$$ at time $t-1$ and postponing consumption for investing in next period's capital stock. The Ramsey-Keynes rule for the Nash equilibrium only internalizes climate change damage occurring domestically since negative climate change externalities to neighboring countries are not taken into account in the shadow price of carbon $\phi_{i, t}^{N E}$.

It is interesting to notice that if a region does not value climate change damages $\left(D_{i}^{\prime}(x)=\right.$ $0 \forall x \geq 0$ ), the Ramsey-Keynes rule boils down to simply: $A_{i, t} F_{i}^{\prime}\left(K_{i, t}\right)-\delta_{K}=\rho_{i}$. This is the golden rule of capital accumulation saying that along an optimal investment path, the net marginal product of capital should be equal to the pure rate of time preference. The fact that there is a production externality causing detrimental climate change in the climate-economy model deflates the marginal return of capital by a factor that depends upon the shadow price of carbon. Therefore, even in a noncooperative Nash equilibrium, the rate of capital accumulation and output growth will be lower in the presence of climate change externalities compared to a BAU scenario without explicit climate change policy.

\subsection{Pareto efficiency}

Pareto efficient consumption, investment and emission abatement paths are obtained as the solution to the following problem: we maximize the utility of one particular country

\footnotetext{
${ }^{5}$ Recall that $\mu_{i, t} \in[0,1]$ has no dimension since it is the fraction of emissions that are abated. $\sigma_{i, t} A_{i, t} F_{i}\left(K_{i, t}^{N E}\right)$ stands for gross carbon emissions without emission abatement $\left(\mu_{i, t}=0\right)$ and is measured in tons of carbon.
} 
(without loss of generality, we will maximize country 1 's utility $W_{1}$ ) subject to the restriction that every other country achieves at least some given ${ }^{6}$ utility level $\bar{W}_{j}$ and subject to the global economy-climate relationships. We also introduce transfers $\Psi_{i}$ (expressed in units of period 0 consumption good) of a once-only nature, with $\Psi_{i}>0(<0)$ denoting a transfer received (paid, respectively). Formally:

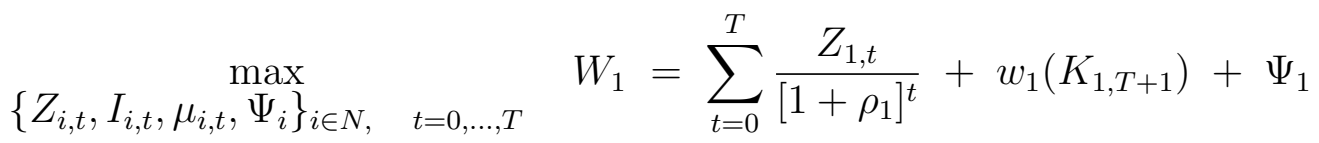

subject to (for all $i \in N$ and for all $0 \leq t \leq T$ ):

$$
\begin{array}{rlrl}
W_{j} & =\sum_{t=0}^{T} \frac{Z_{j, t}}{\left[1+\rho_{j}\right]^{t}}+w_{j}\left(K_{j, T+1}\right)+\Psi_{j} \geq \bar{W}_{j} & & \\
\sum_{j \in N} \Psi_{j} & =0 & & {[\nu]} \\
A_{i, t} F_{i}\left(K_{i, t}\right) \geq Z_{i, t}+I_{i, t}+C_{i}\left(\mu_{i, t}\right)+D_{i}\left(G\left(M_{t}\right)\right) & \forall i, t \quad\left[\zeta_{i, t}\right] \\
K_{i, t+1} & =\left[1-\delta_{K}\right] K_{i, t}+I_{i, t} & \forall i, t \quad\left[\psi_{i, t}\right] \\
M_{t+1} & =\left[1-\delta_{M}\right] M_{t}+\beta \sum_{j \in N} \sigma_{j, t}\left[1-\mu_{j, t}\right] A_{j, t} F_{j}\left(K_{j, t}\right) & \forall t \quad\left[\phi_{t}\right]
\end{array}
$$

with $K_{i, 0}$ and $M_{0}$ given, $\Psi_{i}$ unrestricted in sign, non-negativity constrains on all other variables and $\mu_{i, t} \leq 1$. The Greek symbols between square brackets denote Langrange multipliers for the corresponding constraints. Without loss of generality, we normalize the $\lambda_{i}$ 's such that $\lambda_{1}=1$. The first set of constraints requires that all players other than player 1 enjoy a utility level at least as high as $\bar{W}_{j}$. These constraints combined with the utility maximization for player 1 exhaust all possibilities for Pareto improvements and do therefore lead to a Pareto efficient allocation of resources. The second constraint refers to the transfers that are expressed in period 0 consumption units; the constraint requires that these transfers balance. The other constraints repeat the basic economy and climate relationships, i.e. the individual countries' budget conditions, capital accumulation and carbon concentration accumulation.

Notice that the third constraint (on the countries' budgets) are specified for each $i$ separately: each country is supposed to survive out of its own productive resources only, at each time period $t$, with neither these resources nor the generated consumption good being

\footnotetext{
${ }^{6}$ The given utility levels $\bar{W}_{j}$ for regions $j=2,3, \ldots, n$ should correspond to a feasible allocation in the underlying economy-climate model. By parameterizing these $\bar{W}_{j}$ one can trace out the entire set of Pareto efficient allocations.
} 
transferable to other countries ${ }^{7}$. This is a more restrictive setting than if such transfers were allowed at each $t$ but it is consistent with our deletion of international trade in the RICE model $^{8}$. Thus the solution we compute is actually one of constrained Pareto efficiency. In all what follows, we mean efficient in that sense.

The first-order conditions for all $i \in N$ and $0 \leq t \leq T$ for an interior optimum of (18) resemble strongly the Nash equilibrium first-order conditions stated above. Only condition (13) is fundamentally modified implying that the Pareto efficient stock of carbon in the atmosphere $M_{t}^{*}$ should satisfy instead (the asterisk superscript refers to the values of the variables at the Pareto efficient solution):

$$
\phi_{t-1}^{*}=G^{\prime}\left(M_{t}^{*}\right) \sum_{j \in N} \zeta_{j, t}^{*} D_{j}^{\prime}\left(G\left(M_{t}^{*}\right)\right)+\left[1-\delta_{M}\right] \phi_{t}^{*}, \quad \phi_{T}^{*}=0
$$

Through iterative substitution from (19) the Pareto efficient shadow price of carbon $\phi_{t}^{*}$ is calculated for each $t<T$ in the same way as before. Using this Pareto efficient shadow price of carbon, we obtain the following condition determining the Pareto efficient amount of carbon emission control for country $i$ in period $t$ :

$$
\zeta_{i, t}^{*} \frac{C_{i}^{\prime}\left(\mu_{i, t}^{*}\right)}{\sigma_{i, t} A_{i, t} F_{i}\left(K_{i, t}^{*}\right)}=\beta \phi_{t}^{*}=\beta \sum_{\tau=t+1}^{T}\left[1-\delta_{M}\right]^{\tau-t-1} G^{\prime}\left(M_{\tau}^{*}\right) \sum_{j \in N} \zeta_{j, t}^{*} D_{j}^{\prime}\left(G\left(M_{\tau}^{*}\right)\right)
$$

This expression can be simplified further using the first-order conditions for the variables $Z_{i, t}$ and $\Psi_{i}$. These read:

$$
\zeta_{i, t}^{*}=\frac{\lambda_{i}^{*}}{\left[1+\rho_{i}\right]^{t}} \quad \forall i, t \quad \text { and } \quad \lambda_{i}^{*}=\nu^{*} \quad \forall i
$$

and together they imply for all $i \in N$ :

$$
\zeta_{i, t}^{*}=\frac{\nu^{*}}{\left[1+\rho_{i}\right]^{t}}
$$

Using the latter expression to substitute for the multipliers $\zeta_{i, t}^{*}$ in expression (20) we obtain the Samuelson rule for the Pareto efficient provision of emission reduction:

$$
\frac{1}{\left[1+\rho_{i}\right]^{t}} \frac{C_{i}^{\prime}\left(\mu_{i, t}^{*}\right)}{\sigma_{i, t} A_{i, t} F_{i}\left(K_{i, t}^{*}\right)}=\beta \phi_{t}^{*}=\beta \sum_{\tau=t+1}^{T}\left[1-\delta_{M}\right]^{\tau-t-1} G^{\prime}\left(M_{\tau}^{*}\right) \sum_{j \in N} \frac{D_{j}^{\prime}\left(G\left(M_{\tau}^{*}\right)\right)}{\left[1+\rho_{j}\right]^{\tau}}
$$

\footnotetext{
${ }^{7}$ There should be no confusion between this restriction and the transfers $\Psi_{i}$ introduced earlier: transfers were independent of time (no index $t$ ), in spite of being expressed in units of the consumption good at time zero, and the moment(s) of their implementation was left unspecified. A model with time dependent transfers is proposed in Germain, Toint, Tulkens and De Zeeuw (2003).

${ }^{8}$ While allowing for international trade in capital, Nordhaus and Yang (1996) have to impose severe quantity constraints on the trade flows in order to get realistic results (see their equations A.16-A.18: so severe that the role of trade is virtually annihilated.
} 
The latter condition is a dynamic extension of the traditional optimality rule for static public good models that was first stated by Samuelson (1954). The left hand side (LHS) of the expression stands for the discounted marginal cost for region $i$ of reducing its carbon emissions by an additional ton in period $t$. The RHS consists of the sum from period $t+1$ until the final period $T$ of all regions' discounted future marginal damages from climate change, taking into account the airborne fraction of emissions and the natural decay of atmospheric carbon. In contrast to the Nash equilibrium, the Pareto efficient allocation of emission abatement takes into account total climate change damages affecting all regions in the World (sum of marginal damages over all $j \in N$ ). Thus, the climate externality is fully internalized in Pareto efficient allocations.

Rule (23) does not say that all regions should reduce their emissions in such a way that their discounted marginal abatement costs in each period $t$ be equalized. This would be the case only if all countries had the same discount rate $\rho_{i}=\rho$. Our Samuelson rule is instead a weighted extension of the traditional optimality rule for the provision of public goods. Countries characterized by a high discount rate are required to perform relatively more emission abatement since their opportunity cost of an additional $\$$ of consumption is lower. For a more precise treatment of this argument and the trade off between equity and efficiency, see, e.g., Eyckmans, Proost and Schokkaert (1993).

It is also interesting to note that the allocation of abatement efforts does not depend on the vector of given utility levels $\bar{W}_{j}$. Changing this vector of utilities for regions 2 to $n$ will modify the values of the optimal transfers $\Psi_{i}^{*}$ and the shadow price $\nu^{*}$, but it does not alter the Samuelson condition (23). The allocation of abatement is therefore independent of the allocation of transfers ${ }^{9}$. This is an important property for the rest of the paper since we will introduce in section 4 a particular transfer scheme to deal with participation constraints. Whatever these transfers will look like, they will not alter the allocation of abatement according to condition (23).

Finally, we derive the condition for the Pareto efficient accumulation of capital in the presence of an environmental externality:

$$
\rho_{i}+\delta_{K}=A_{i, t} F_{i}^{\prime}\left(K_{i, t}^{*}\right)\left[1-\beta \sigma_{i, t}\left[1-\mu_{i, t}^{*}\right]\left[1+\rho_{i}\right]^{t} \phi_{t}^{*}\right]
$$

Though this condition looks exactly the same as condition (17), it is fundamentally different since in the Pareto efficient case, the shadow price of carbon $\phi_{t}^{*}$ internalizes all climate change externalities whereas it only internalizes domestic damages in the Nash equilibrium.

\footnotetext{
${ }^{9}$ Since we have assumed linear utility functions for all players, the Pareto efficient allocation of abatement efforts $\left(\mu_{i, t}^{*}\right)_{i \in N ; t=0,1, \ldots, T}$ can be characterized also as the solution of an unweighted sum of all the players welfare: $\max \sum_{j \in N} W_{j}$. We will use this formulation in section 3.3 to solve the abatement choice problem of any coalition $S \subset N$.
} 


\subsection{Partial agreement Nash equilibria w.r.t. a coalition}

The previous two sections described two extreme cases as to cooperation. In a Pareto efficient scenario, all regions take action jointly to reduce their emissions of carbon dioxide and they do so by internalizing completely the external effects of their carbon emissions. In the Nash equilibrium, every region reduces its carbon emissions also but to a lesser extent because they only internalize the external effects of their emissions that affect their own territory. Intermediate cases are conceivable, when only some subgroup of regions agrees to coordinate its emission reduction policies ${ }^{10}$.

In order to characterize this situation of partial cooperation, we use the concept of partial agreement Nash equilibrium w.r.t. a coalition (PANE), introduced by CT (1995 and 1997)(CT hereafter). Suppose a coalition $S \subseteq N$ forms with $s$ members. In a partial agreement Nash equilibrium w.r.t. coalition $S$, this coalition chooses actions that are most beneficial from the group's point of view while the outsiders to the coalition choose actions that maximize their individual utility. The PANE w.r.t. $S$ can be interpreted as a special type of Nash equilibrium in which a coalition $S$ coordinates its policies taking as given the emission strategies of the outsiders who, in turn, are playing a non-cooperative Nash strategy against $S$. Formally, a partial agreement Nash equilibrium w.r.t. coalition $S$ is a combination of strategies that solves simultaneously the following $n-s+1$ maximization problems:

$$
\begin{array}{ll}
\text { for the insiders }(i \in S): & \max \quad W_{S} \stackrel{\text { def }}{=} \sum_{i \in S} W_{i}=\sum_{i \in S} \sum_{t=0}^{T} \frac{Z_{i, t}}{\left[1+\rho_{i}\right]^{t}} \\
\text { and for each outsider }(i \in N \backslash S): \quad \max \quad W_{i}=\sum_{t=0}^{T} \frac{Z_{i, t}}{\left[1+\rho_{i}\right]^{t}}
\end{array}
$$

subject to the individual resource constraints (1), the production function (2), the capital accumulation conditions (3), the definition of carbon emissions (4), carbon concentration (5) and temperature change (6) equations being satisfied. This concept encompasses both those of Pareto efficiency (for $S=N$ ) and of a Nash Equilibrium (for $S=\{i\}$ for some $i \in N)$.

First-order conditions for a PANE w.r.t. a coalition $S$ can be derived in the same way as before. These first-order conditions appear as a mixture of the first-order conditions for a

\footnotetext{
${ }^{10}$ The 1997 Kyoto Protocol on greenhouse gases emission reduction may be seen as an example of such partial cooperation. However, this view was not shared by Chander, Tulkens, van Ypersele and Willems (2002) who proposed instead an interpretation in terms of an all players coalition. Indeed, although many developing countries have not assumed quantified emission ceilings in the 1997 Kyoto Protocol, they are official signatories of it. Now, the ratification stage that followed the withdrawal of the US and a few other countries while all other countries ratify, brings one back to a situation of PANE w.r.t. the ratifying coalition.
} 
Pareto efficient allocation and a Nash equilibrium. For the outsiders, first-order conditions are exactly similar to the conditions (16) and (17). Indeed, outsiders take into account only domestic climate change damages. As a limiting case, for $S=\{i\}$ for some $i \in N$, all the outsiders' conditions coincide exactly with (16) and (17) respectively.

For the insiders of $S$, first-order conditions look very similar like the conditions (20) and (24) except for the fact that the summation of marginal damages bears only upon the members

of coalition $S$. Insiders internalize the negative externalities from climate change only among themselves:

$$
\begin{aligned}
\frac{1}{\left[1+\rho_{i}\right]^{t}} \frac{C_{i}^{\prime}\left(\mu_{i, t}^{S}\right)}{\sigma_{i, t} A_{i, t} F_{i}\left(K_{i, t}^{S}\right)} & =\beta \sum_{\tau=t+1}^{T}\left[1-\delta_{M}\right]^{\tau-t-1} G^{\prime}\left(M_{\tau}^{S}\right) \sum_{j \in S} \frac{D_{j}^{\prime}\left(G\left(M_{\tau}^{S}\right)\right)}{\left[1+\rho_{j}\right]^{\tau}} \\
\rho_{i}+\delta_{k} & =A_{i, t} F_{i}^{\prime}\left(K_{i, t}^{S}\right)\left[1-\beta \sigma_{i, t}\left[1-\mu_{i, t}^{S}\right]\left[1+\rho_{i}\right]^{t} \phi_{S, t}^{S}\right]
\end{aligned}
$$

where $\phi_{S, t}^{S}$ stands for the shadow price of the atmospheric carbon concentration for coalition $S$. It is given by the RHS of expression (27). For $S=N$ the insiders' conditions reduce to $(20)$ and (24) respectively and the PANE w.r.t. $N$ coincides with the Pareto efficient allocation.

\section{Transfers ensuring individual and coalititional ra- tionality}

\subsection{Individual and coalitional rationality}

As is well known, the fact that a particular allocation is Pareto efficient does not imply that all regions are better off compared to a Nash equilibrium. While many regions are net winners, some other regions may be net losers. And if a region is worse off, it will not accept an agreement that proposes to implement such an allocation. In this case we will say that the proposed agreement does not satisfy individual rationality.

Not only individual regions may be worse off under the Pareto efficient solution: also coalitions of two or more regions may find out that they can do better if the joint payoff of their members in the partial agreement Nash equilibrium is higher than at the efficient allocation. If this is the case, we say that the efficient allocation does not satisfy coalitional rationality.

Allocations that satisfy both individual rationality for all regions, and coalitional rationality for all possible coalitions of the regions are said to belong to the "core" of a cooperative 
game associated with the economic model under consideration. In the present case of the CWS model, the players of the game are the regions, and the players' strategies are the emission abatement policies chosen by the regions. In this setting, the core property of an allocation thus ensures that no coalition $S$ could be better off by proposing a partial agreement Nash equilibrium w.r.t. itself. This property can be interpreted as a necessary (though not sufficient) condition for a voluntary international agreement to be sustained, as argued in Tulkens (1998).

\subsection{The transfers formula}

These considerations imply that a climate treaty implementing the Pareto efficient allocation prescribed by the Samuelson rule (20) may not emerge as a voluntary agreement among the emitters of carbon dioxide. However, transfers of consumption offer a way to induce such voluntary cooperation. In particular, we consider in this paper the transfer scheme proposed by GTT for stock pollution problems. In this section we present a reinterpretation of this transfer scheme for the CWS model. We start from a Pareto efficient allocation of emission abatement efforts that solves the Samuelson conditions (20) for all $i$ and $0 \leq t \leq T$, and we then modify this allocation by introducing transfers of the consumption good defined as follows.

Let $W_{i}^{N E}$ be the discounted consumption stream of region $i$ under the Nash equilibrium and $W_{i}^{*}$ as the discounted consumption stream of region $i$ in the Pareto efficient outcome:

$$
W_{i}^{N E}=\sum_{t=0}^{T} \frac{Z_{i, t}^{N E}}{\left[1+\rho_{i}\right]^{t}} \quad \text { and } \quad W_{i}^{*}=\sum_{t=0}^{T} \frac{Z_{i, t}^{*}}{\left[1+\rho_{i}\right]^{t}}
$$

GTT suggested the following transfer of the consumption good (with shares $\pi_{i} \geq 0$ such that $\left.\sum_{i} \pi_{i}=1\right)$ :

$$
\Psi_{i}=-\left[W_{i}^{*}-W_{i}^{N E}\right]+\pi_{i}\left[\sum_{j \in N} W_{j}^{*}-\sum_{j \in N} W_{j}^{N E}\right]
$$

This transfer formula takes away, from every region, the difference between its Pareto efficient consumption allocation $W_{i}^{*}$ and its consumption level $W_{i}^{N E}$ at the Nash equilibrium; moreover, it divides the surplus of cooperation over non-cooperation in proportion to the weights $\pi_{i}$. These weights $\pi_{i}$ are equal to the ratios $D_{i}^{\prime} / \sum_{j} D_{j}^{\prime}$. Regions with a relatively high share $\pi_{i}$ get relatively more of the surplus. The transfer scheme then yields the following consumption level for each $i \in N$ :

$$
W_{i}^{*}+\Psi_{i}=W_{i}^{N E}+\pi_{i}\left[\sum_{j \in N} W_{j}^{*}-\sum_{j \in N} W_{j}^{N E}\right] \geq W_{i}^{N E}
$$


Clearly, the resulting consumption allocation is preferred over the Nash equilibrium allocation $W_{i}^{N E}$ by all $i \in N$ as long as there is a positive surplus to cooperation. Hence, the allocation with transfers is always individually rational.

Moreover, GTT have shown that the transfer scheme gives rise to an allocation of consumption which belongs to the core of the cooperative emission abatement game, provided that damage cost functions are linear. However, damage functions in the CWS model are nonlinear, implying that it is not sure whether the core property still holds. Nevertheless we can experiment numerically with the transfer formula (29) and check by means of simulations whether or not, with the transfers (29), coalitions have an interest in forming.

With nonlinear damage cost functions $D_{i}\left(\Delta T_{t}\right)$ the $\pi_{i}$ 's in the transfer formula (29) are no longer constant over time. In order to take that into account we generalize the ratios $\pi_{i}$ by introducing $\widetilde{\pi}_{i}$ defined in the following way:

$$
\tilde{\pi}_{i}=\frac{\sum_{0 \leq t \leq T} \frac{D_{i}^{\prime}\left(\Delta T_{t}^{*}\right)}{\left[1+\rho_{i}\right]^{t}}}{\sum_{j \in N} \sum_{0 \leq t \leq T} \frac{D_{j}^{\prime}\left(\Delta T_{t}^{*}\right)}{\left[1+\rho_{j}\right]^{t}}},
$$

that is, the share of region $i$ in the aggregate discounted World marginal climate change damage costs. With these generalized ratios $\widetilde{\pi}_{i}$ introduced in expression (29, we obtain new transfers $\widetilde{\Psi}_{i}$ that we will call generalized GTT transfers:

$$
\widetilde{\Psi}_{i}=-\left[W_{i}^{*}-W_{i}^{N E}\right]+\widetilde{\pi}_{i}\left[\sum_{j \in N} W_{j}^{*}-\sum_{j \in N} W_{j}^{N E}\right]
$$

\subsection{Checking for the core property}

To check whether the Pareto efficient allocation, supplemented with generalized GTT transfers is a core allocation, it is sufficient to check that the following inequalities hold for all coalitions of $S \subseteq N$ :

$$
W_{S}^{S} \stackrel{\text { def }}{=} \sum_{i \in S} W_{i}^{S} \leq \sum_{i \in S}\left[W_{i}^{*}+\widetilde{\Psi}_{i}\right] \stackrel{\text { def }}{=} W_{S}^{*}+\widetilde{\Psi}_{S} \quad \forall S \subseteq N
$$

Indeed, when $W_{S}^{*}+\widetilde{\Psi}_{S} \geq W_{S}^{S}$, the coalition $S$ obtains a higher payoff under the transfer scheme than it would obtain at the Partial Agreement Nash equilibrium w.r.t. itself; thus, the corresponding coalitional rationality constraint is satisfied. 


\section{Simulations}

\subsection{Numerical specification of the CWS model}

From the general specification (1)-(6) of the CWS model we now move to a numerical specification, suitable for simulations to test the "core" property just discussed. The temperature change equation (function $G$ in (6)) is taken from the the climate-economy model RICE by Nordhaus and Yang (1996) as well as most of the parameter values and all basic data on GDP, population, capital stock, carbon emissions and concentration and global mean temperature. A complete list of the equations and of the parameter values that we use, is provided in the appendix, see (32)-(42). The simulation model partitions the World into six regions: USA, Japan (JPN), European Union (EU), China (CHN), Former Soviet Union (FSU) and Rest Of the World (ROW).

However, contrary to Nordhaus and Yang (1996), we select different parameter values on two crucial points. First, we choose a discount rate of $1.5 \%$ for the industrialized regions ( $U S A, J P N, E U$ and $F S U$ ) and a higher discount rate of $3 \%$ for the developing regions $C H N$ and $R O W$. This difference reflects taste differences across regions concerning the priority of economic development over environmental concern. Secondly, we increase the value of the exponent in the climate change damage function (36) from 1.5 to 2.0. This choice was made after learning from our colleagues climatologists that temperature changes of $7^{\circ}$ Celsius or more are to be considered as catastrophic ones: indeed, this difference is larger than the change in temperature between the last Ice Age and current temperature. Finally, we also revised downward the projected exogenous technology growth for FSU in order to match more closely current predictions on economic production for this region.

\subsection{Computing equilibria}

In order to calculate partial agreement Nash equilibria w.r.t. coalitions (including plain Nash equilibria), we use a standard numerical algorithm to compute non-cooperative Nash equilibria where the coalition $S$ is treated as one player in the emission game. In every iteration, a strategy is determined for each player, consisting of an investment and emission abatement path that maximizes its life time utility given the strategies of the other players. This iteration process is continued until the Euclidean distance between the strategy vectors in two consecutive iterations is smaller than a given threshold value.

As perfect information is assumed, the resulting equilibrium is an open-loop equilibrium. The algorithm is equivalent to the one used by Yang (1998) to calculate numerically so-called "hybrid" coalition solutions. It was implemented using the optimization software GAMS. With 32 periods (decades), solving for one partial agreement Nash equilib- 
rium only takes a couple of minutes on a Pentium 4, 1 Ghz $\mathrm{PC}^{11}$. Theoretically, a sufficient condition for convergence of this kind of algorithm is that the absolute value of the slope of the reaction functions of all players be smaller than one. In that case, the reaction mapping is a contraction and convergence is assured. In the CWS model (and in RICE) this condition on the slope of the reaction functions is not easy to check because of the dynamic specification of the carbon cycle and climate change model. In practice however, we never encountered any convergence problem during the numerous simulations. We never found multiple equilibria by changing the set of starting values.

\subsection{Reference simulations: BAU, NASH, EFF}

\subsubsection{Carbon emissions}

Figure 1 shows annual World carbon emissions in three scenarios: business-as-usual (BAU), Nash equilibrium (NASH) and Pareto efficiency without transfers (EFF) ${ }^{12}$. We only consider carbon emissions originating from fossil fuel use. World carbon emissions in 1990 amount to approximately 6 gigatons ${ }^{13}$ of carbon.

In the BAU scenario, we assume that countries do not value climate change and do nothing to restrict their carbon emissions, i.e. $\mu_{i, t}=0$ for all $i$ and $t$. BAU emissions grow continuously to reach nearly $40 \mathrm{GtC}$ by the year 2100 and more than $62 \mathrm{GtC}$ in 2200 . BAU emissions continue to grow throughout the entire time horizon although the pace of growth gradually slows down.

In the NASH equilibrium scenario, emissions grow at a slightly slower rate to reach about $38 \mathrm{GtC}$ by the year 2100 and $59 \mathrm{GtC}$ by 2200. Also in NASH, emissions continue to grow though growth decelerates.

Pareto efficient carbon emissions (EFF) are substantially lower than BAU and NASH emissions: by the year 2100 they amount to some $24 \mathrm{GtC}$, and only $21 \mathrm{GtC}$ by 2200 . This is about half the BAU emission level in 2100 and almost one third of BAU emissions in 2200. In contrast to BAU and NASH emissions, the EFF emission path rises until 2150, levels off at about $26 \mathrm{GtC}$ and decreases afterwards.

\footnotetext{
${ }^{11}$ All data and simulation programs are available from the authors upon request.

${ }^{12}$ All figures report data for a time horizon of 2000 to 2250 . However, all calculations were made until the year 3200 so as to avoid distortions from end period effects on the period we are interested in.

${ }^{13}$ All carbon emission and concentration data will be expressed in gigatons of carbon (GtC) which is the same as billion tons of carbon (btC), i.e. $10^{9}$ tons of carbon.
} 
Figure 1: World carbon emissions (GtC)

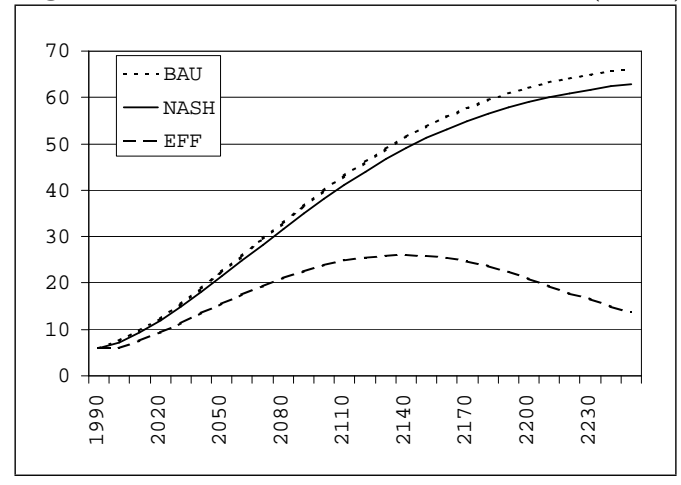

Figure 2: World carbon concentration (GtC)

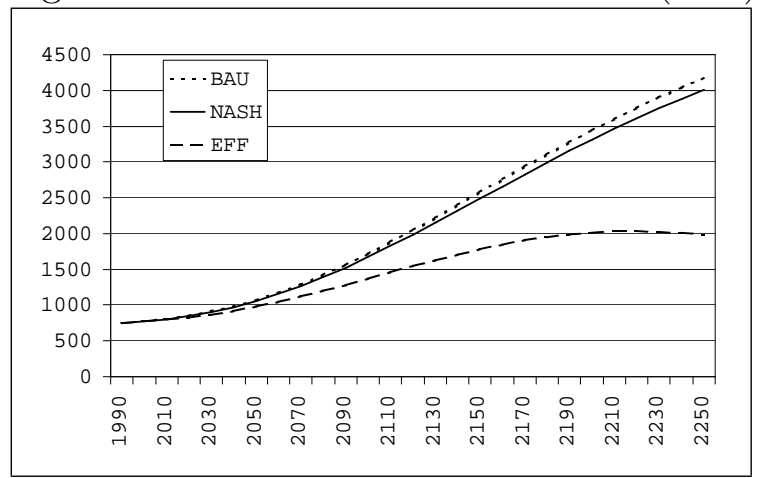

\subsubsection{Atmospheric carbon concentrations}

Figure 2 shows the atmospheric carbon concentration in the BAU, NASH equilibrium and EFF scenarios respectively. 1990 atmospheric carbon concentration amounted approximately $750 \mathrm{GtC}$. Under BAU, the atmospheric carbon concentration rises steadily and reaches about $1718 \mathrm{GtC}$ in 2100 and $3443 \mathrm{GtC}$ in 2200. Doubling of the concentration w.r.t. 1990 takes place between 2080 and 2090. The NASH carbon concentration path follows closely the BAU path and continues to grow steadily all over the time horizon.

By contrast, in the EFF scenario, atmospheric carbon concentrations grow at a much slower rate and reach 1368 GtC in 2100 and 2017 GtC in 2200. Doubling of the atmospheric carbon concentration w.r.t. 1990 is postponed until somewhere between 2110 and 2120. The carbon concentration levels off at about $2000 \mathrm{GtC}$ by the year 2200 . In that respect, the Pareto efficient outcome can be considered more sustainable than the BAU and NASH scenarios.

\subsubsection{Temperature changes}

Figure 3 shows the temperature increase compared to preindustrial times for the three reference scenarios. By the year 2100 temperature rises with 2.77, 2.70 and $2.24^{\circ}$ Celsius in the BAU, NASH and EFF scenarios respectively. By the year 2200, differences are more pronounced: 5.42, 5.30 and $3.93^{\circ}$ Celsius. Whereas BAU and NASH temperatures continue to rise steadily, the Pareto efficient temperature change levels off at about $4^{\circ}$ Celsius by the end of the time horizon shown in Figure 3. This occurs only about 50 years after the atmospheric carbon concentration has levelled off because of the long time inertia of the climate system. 
Figure 3: Global mean temperature change $\left({ }^{\circ}\right.$ Celsius)

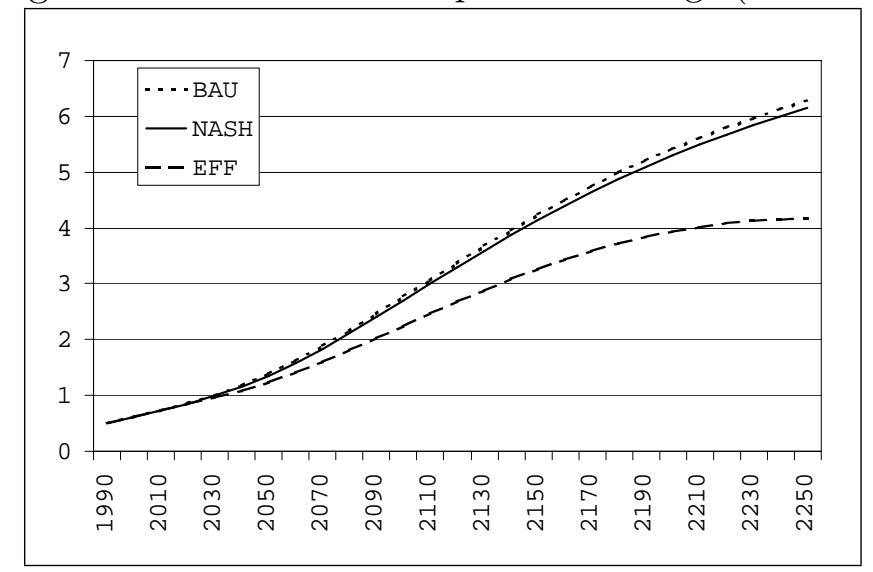

\subsubsection{Emission control rates}

Figure 4 shows the time path of emissions control $\left(\mu_{i, t}^{N E}\right)$ for the NASH equilibrium. There are substantial differences across regions. Taking averages of abatement effort over time, we see that $C H N$ produces the highest abatement level (about $7.70 \%$ ), followed by $E U$ with $7.24 \%$ and $U S A$ with $6.44 \%$. The lowest abatement effort is by $R O W$ with only $1.45 \%$. World average abatement amounts to $3.74 \%$. The time path of emissions control rate of $R O W$ lies far below the paths of the other regions due to strong free riding incentives within this heterogeneous region ${ }^{14}$. $C H N$ and $E U$ are situated at the other end of the spectrum. For $C H N$ this is due to the combination of low emission abatement costs and substantial climate change damages. For $E U$ this is due to their relatively high climate change damage valuation.

Finally, Figure 5 shows the time path of emission control rates $\left(\mu_{i, t}^{*}\right)$ for the Pareto efficient scenario EFF. Average World emission abatement w.r.t. BAU emissions rises from 3.74\% in BAU to about $37.14 \%$ in EFF. In EFF, both $C H N$ and $R O W$ should reduce their emissions substantially more than the others regions (68.13\% and $55.50 \%$ respectively) and, more strikingly, their abatement effort rises over time. This last fact is due to the higher discount rates of $C H N$ and $R O W$. Since they value the future less than the other regions, they are asked to perform ever more effort as time goes by. For them, the opportunity cost of forgoing an additional $\$$ of consumption is valued less than for industrialized regions, cfr. formula (20).

\footnotetext{
${ }^{14}$ As in Nordhaus and Yang (1996) we model free riding behavior in the $R O W$ region by revising downward their climate change damage parameter in all non-cooperative scenario in which $R O W$ is standing on its own. Without this modification, non-cooperative emission control rate by $R O W$ is unrealistic because of its size.
} 
Figure 4: NASH emissions abatement (\%)

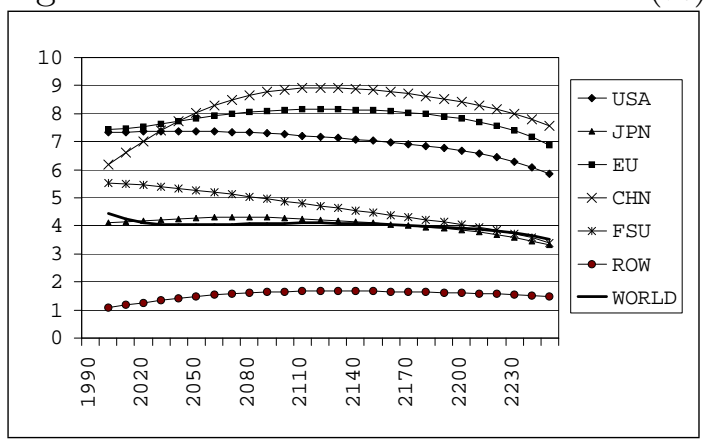

Figure 5: EFF emissions abatement (\%)

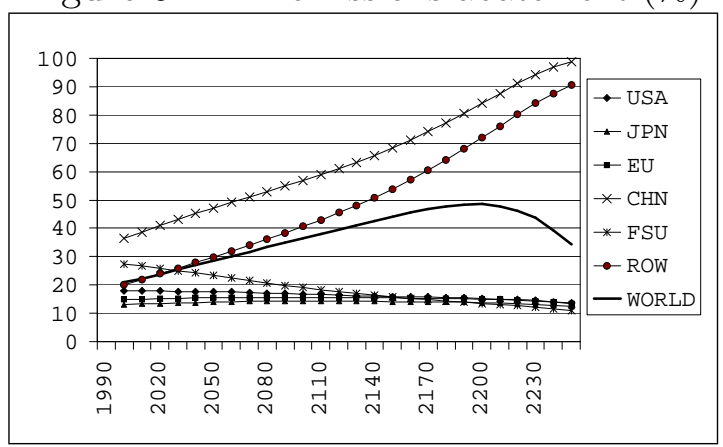

\subsubsection{Macroeconomic magnitudes}

Figures 6 and 7 exhibit the time profiles of aggregate output $(Y)$, consumption $(Z)$, investment $(I)$, abatement costs $(C)$ and damage costs $(D)$ of the BAU and EFF scenarios (in the NASH scenario, these magnitudes are indistinguishably close to the BAU values). One first notices that output growth is fairly similar, as well as the consumption profile (but see below). The small differences between both scenarios stem from the way in which the difference $Y-Z$ is allocated: the EFF scenario entails much less damage costs $D$, but this is only obtained from devoting part of the production to emission abatement (measured by $C$ ), with investment $(I)$ not observably affected by this substitution.

Figure 6: Composition GDP in BAU (\% of GDP)

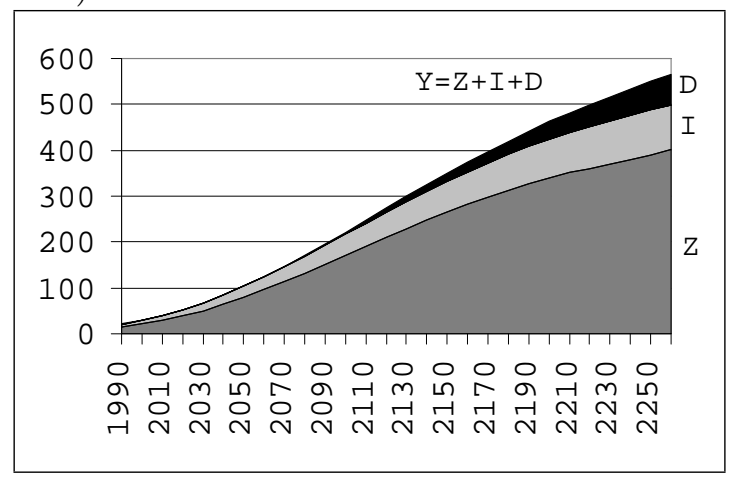

Figure 7: Composition GDP in EFF (\% of GDP)

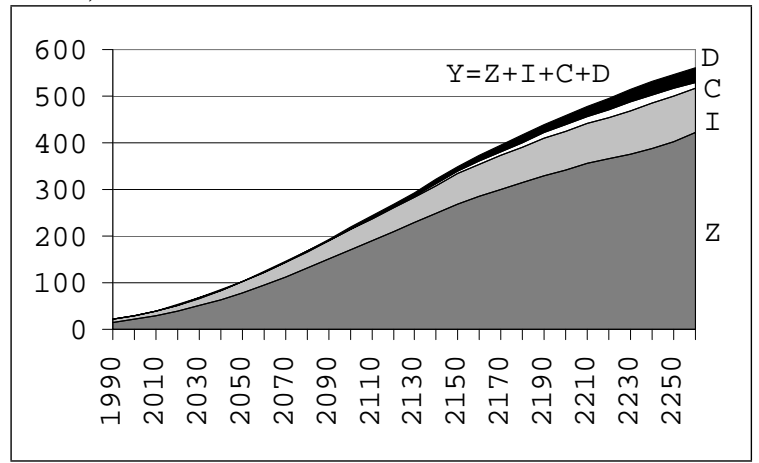


Figure 8: Abatement and damage costs (\% of GDP)

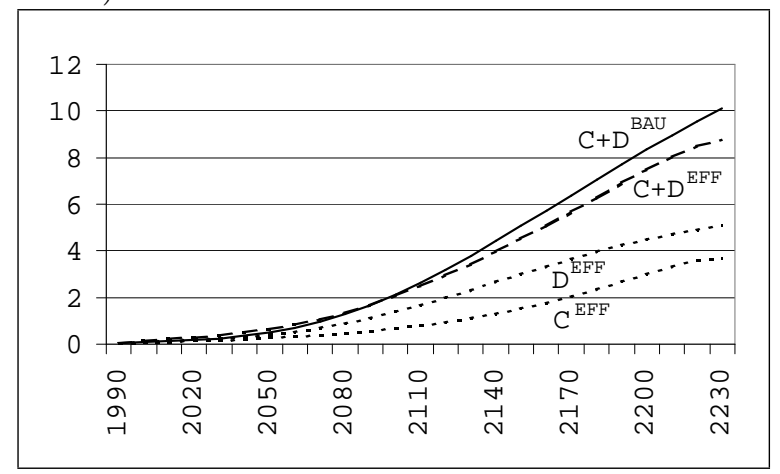

\subsection{On the value of cooperation over non-cooperation}

Figure 8 offers a closer look at the evolution of abatement damage costs for the extreme scenarios BAU and EFF. In the former scenario, where no emission abatement occurs, climate change damages rise to more than $10 \%$ of GDP by 2250 . On the other hand, in the EFF scenario, where the abatement effort goes to $4 \%$ of GDP by 2250, damage costs nevertheless rise to about $5 \%$ by the end of the period considered. Taken together abatement costs and damage costs, the EFF scenario yields a better result than the BAU scenario, the difference amounts to approximately $1 \%$ of World GDP by 2250 . In this sense, we can conclude that the EFF scenario is more sustainable than the BAU scenario.

Table 1: World discounted consumption over 320 years (billion 1990US\$ and \%)

\begin{tabular}{l|rrrrrr}
\hline scenario & BAU & NASH & $\frac{\text { NASH }}{\text { BAU }}$ & EFF & $\frac{\text { EFF } \%}{\text { BAU }}$ & $\frac{\text { EFF }}{\text { NASH }}$ \\
\hline USA & 78,270 & 78,353 & 0.11 & 78,986 & 0.91 & 0.81 \\
Japan & 42,867 & 42,909 & 0.10 & 43,222 & 0.83 & 0.73 \\
EU & 102,612 & 102,731 & 0.12 & 103,650 & 1.01 & 0.89 \\
China & 9,131 & 9,141 & 0.11 & 8,862 & -2.95 & -3.06 \\
FSU & 23,763 & 23,794 & 0.13 & 24,025 & 1.10 & 0.97 \\
ROW & 81,049 & 81,137 & 0.11 & 81,093 & 0.05 & -0.05 \\
\hline World & 337,692 & 338,065 & 0.11 & 339,837 & 0.64 & 0.52 \\
\hline
\end{tabular}

The latter conclusion is confirmed by Table 1 which summarizes total discounted consumption (over the entire horizon of the computation, i.e. 320 years) for each region under the alternative cooperation scenarios. The last row World reveals the overall magnitudes at stake. Discounted consumption amounts to 337,692 (BAU), 338,065 (NASH) and 
339,837 (EFF) billion $\$$ respectively. The gain at the World level between the BAU and $\mathrm{NASH}$ is rather small $(+0.11 \%)$, but the additional gain obtained by moving from NASH to $\mathrm{EFF}$ is more important $(+0.52 \%)$. Overall, the welfare gain of moving from the BAU to the EFF scenario is about $0.64 \%$.

How do these figures relate to the original RICE model as reported in Table 4, p.757 in Nordhaus and Yang (1996)? The overall difference is much larger in our calculations than in RICE. The difference between BAU and EFF amounts to 1772 billion $\$$ in Table 1 against only 344 billion $\$$ in Nordhaus and Yang (1996). However, this comparison is not quite correct because we are using different discount rates and a different time horizon (320 years). In particular, we use 1.5\% for industrialized regions and 3.0\% for developing regions whereas Nordhaus and Yang (1996) use "region-specific discount rates on consumption [...that] average about $4.5 \%$ per year (p.755, italics added). Clearly, through our choice of discount rates we value climate change damages avoided in the far future much more heavily than Nordhaus and Yang (1996). Moreover, we use a more convex damage function. Overall, our value of cooperation must be considerably higher than the one calculated by RICE.

The most striking difference is the pattern of winners and losers in the EFF scenario compared to NASH. Whereas Nordhaus and Yang (1996) find that USA is the only loser, we find that both $C H N$ and $R O W$ would lose from joining an efficient agreement without transfers. Again, it is difficult to compare this pattern with RICE since we have no trade in our cooperative solution. Unfortunately, Nordhaus and Yang (1996) did not report the trade flows in their efficient (Negishi) solution so we cannot judge whether the difference in the pattern of winners and losers is due to the absence of trade flows in our model.

Though there is only an increase of total World consumption of about $0.5 \%$ between NASH and EFF, emission abatement policies do differ substantially between both scenarios as it appears from Figures 4 and 5. Similarly, Figure 3 shows importantly smaller temperature changes under EFF than under both BAU and NASH. Thus, while internationally coordinated policies appear to be importantly different from uncoordinated ones as far as environmental variables are concerned, the present model reveals that there may be some economic indifference between them ${ }^{15}$.

\footnotetext{
${ }^{15}$ This last feature leads some authors, e.g. Hackl and Pruckner (2002), to minimize the importance of cooperative policies. Our findings concerning abatement and temperature change suggest that from an environmental point of view, optimality does matter a lot. Our point in the next section below on how to achieve it cooperatively becomes all the more important.
} 


\subsection{Checking the core property of the Pareto efficient scenario without transfers}

Concerning individual rationality, we observe from Table 1 that both China and $R O W$ are experiencing a loss in lifetime consumption from moving from the Nash equilibrium to the Pareto efficient solution. This is not surprising since both regions are characterized by relatively low marginal emission reduction costs and/or relatively low marginal willingness to pay for environmental quality. From a global efficiency point of view, it might be desirable to require a substantial contribution from low cost regions in the global emission abatement effort. However the value of the avoided climate change damage might be insufficient for these regions to compensate for their high abatement effort.

In order to check for coalitional rationality, i.e. whether there exist groups of regions that would benefit more from forming a coalition of their own than from the Pareto efficient allocation, we calculated all possible partial agreement Nash equilibria for the CWS model. The number of all possible coalitions is given by $2^{\# N}=64$ for 6 regions. A complete list of all coalition values and their payoffs is given in Table 2 .

The entries in Table 2 have been sorted in increasing order of coalition size. The first six lines refer to the payoff of the individual countries in the Nash equilibrium. The next 15 lines refer to all pairs, the next 20 lines to coalitions of size three and so on. The last line refers the Pareto efficient allocation where $S=N$. Payoff figures are reported in billion 1990US\$.

The first column contains a six digit key from which the structure of the coalition can be deducted. If a region is a member of the coalition, it obtains a "1" at the appropriate position in the key. For instance, the key "111111" refers to $S=N=\{U S A, J P N, E C, C H N$, $F S U, R O W\}$, "100000" refers to $S=\{U S A\}$, "111000" refers to $S=\{U S A, J P N, E C\}$ and so on.

Column two $\left(W_{S}^{S}=\sum_{S} \sum_{t} W_{j, t}^{S} /\left[1+\rho_{j}\right]^{t}\right)$ contains the value of a coalition under its corresponding partial agreement Nash equilibrium. Column three $\left(W_{S}^{*}=\sum_{S} \sum_{t} W_{j, t}^{*} /[1+\right.$ $\left.\rho_{j}\right]^{t}$ ) contains the total of what the members of each coalition get in the Pareto efficient allocation. Columns four and five show the difference between $W_{S}^{*}$ and $W_{S}^{S}$ in absolute amounts and in percentages.

The differences $W_{S}^{*}-W_{S}^{S}$ measure the gain, for each coalition $S$, from accepting the Pareto efficient allocation rather than sticking to a partial agreement Nash equilibrium w.r.t. itself. If this difference is negative, it means that $S$ is worse off at the EFF allocation and that the voluntary participation constraint for coalition $S$ is not satisfied. There are twelve coalitions with $\# S \geq 2$ for which the voluntary participation constraint is violated. In particular many coalitions containing $C H N$ and $R O W$ are in this situation. 
Table 2: Coalitions payoffs at all PANE w.r.t. a coalition $\left(W_{S}^{S}\right)$ and at EFF $\left(W_{S}^{*}\right)$; generalized GTT transfers $\left(\widetilde{\Psi}_{S}\right)$ (billion 1990US\$)

\begin{tabular}{|c|c|c|c|c|c|c|c|c|}
\hline key & $W_{S}^{S}$ & $W_{S}^{*}$ & $W_{S}^{*}-W_{S}^{S}$ & $(\%)$ & $\widetilde{\Psi}_{S}$ & $W_{S}^{*}+\widetilde{\Psi}_{S}$ & $W_{S}^{*}+\widetilde{\Psi}_{S}-W_{S}^{S}$ & $(\%)$ \\
\hline \multicolumn{9}{|c|}{ coalitions of 1 country } \\
\hline 100000 & 78353 & 78986 & 633 & 0.808 & -282 & 78704 & 351 & 0.448 \\
\hline 010000 & 42909 & 43222 & 313 & 0.729 & -121 & 43102 & 192 & 0.448 \\
\hline 001000 & 102731 & 103650 & 919 & 0.895 & -423 & 103226 & 496 & 0.482 \\
\hline 000100 & 9141 & 8862 & -279 & -3.057 & 333 & 9195 & 54 & 0.591 \\
\hline 000010 & 23794 & 24025 & 231 & 0.969 & -123 & 23902 & 108 & 0.452 \\
\hline 000001 & 81137 & 81093 & -44 & -0.054 & 616 & 81709 & 572 & 0.705 \\
\hline \multicolumn{9}{|c|}{ coalitions of 2 countries } \\
\hline 110000 & 121264 & 122208 & 945 & 0.779 & -403 & 121806 & 542 & 0.447 \\
\hline 101000 & 181090 & 182636 & 1546 & 0.854 & -706 & 181930 & 841 & 0.464 \\
\hline 100100 & 87535 & 87848 & 312 & 0.357 & 51 & 87899 & 364 & 0.416 \\
\hline 100010 & 102151 & 103011 & 860 & 0.842 & -405 & 102605 & 455 & 0.445 \\
\hline 100001 & 159829 & 160079 & 250 & 0.156 & 334 & 160413 & 584 & 0.365 \\
\hline 011000 & 145642 & 146872 & 1230 & 0.845 & -544 & 146328 & 686 & 0.471 \\
\hline 010100 & 52062 & 52084 & 22 & 0.043 & 213 & 52297 & 235 & 0.451 \\
\hline 010010 & 66705 & 67247 & 542 & 0.813 & -244 & 67003 & 299 & 0.448 \\
\hline 010001 & 124262 & 124315 & 53 & 0.043 & 495 & 124811 & 548 & 0.441 \\
\hline 001100 & 111946 & 112511 & 566 & 0.505 & -90 & 112421 & 476 & 0.425 \\
\hline 001010 & 126531 & 127674 & 1143 & 0.903 & -546 & 127128 & 597 & 0.471 \\
\hline 001001 & 184315 & 184743 & 427 & 0.232 & 192 & 184935 & 620 & 0.336 \\
\hline 000110 & 32944 & 32886 & -58 & -0.175 & 210 & 33097 & 153 & 0.463 \\
\hline 000101 & 90467 & 89955 & -512 & -0.566 & 949 & 90904 & 437 & 0.483 \\
\hline 000011 & 105134 & 105118 & -17 & -0.016 & 493 & 105610 & 476 & 0.453 \\
\hline \multicolumn{9}{|c|}{ coalitions of 3 countries } \\
\hline 111000 & 224007 & 225858 & 1851 & 0.826 & -826 & 225032 & 1024 & 0.457 \\
\hline 110100 & 130486 & 131070 & 584 & 0.448 & -69 & 131001 & 515 & 0.394 \\
\hline 110010 & 145067 & 146233 & 1166 & 0.804 & -526 & 145707 & 641 & 0.442 \\
\hline 110001 & 202879 & 203301 & 422 & 0.208 & 213 & 203514 & 635 & 0.313 \\
\hline 101100 & 190415 & 191497 & 1083 & 0.569 & -372 & 191125 & 711 & 0.373 \\
\hline 101010 & 204903 & 206660 & 1757 & 0.857 & -829 & 205832 & 928 & 0.453 \\
\hline 101001 & 263009 & 263729 & 719 & 0.274 & -90 & 263639 & 630 & 0.239 \\
\hline 100110 & 111367 & 111872 & 505 & 0.453 & -72 & 111800 & 433 & 0.389 \\
\hline 100101 & 169139 & 168941 & -199 & -0.117 & 667 & 169608 & 468 & 0.277 \\
\hline 100011 & 183752 & 184103 & 352 & 0.191 & 211 & 184314 & 562 & 0.306 \\
\hline 011100 & 154905 & 155734 & 829 & 0.535 & -211 & 155523 & 618 & 0.399 \\
\hline 011010 & 169448 & 170897 & 1448 & 0.855 & -667 & 170230 & 781 & 0.461 \\
\hline 011001 & 227376 & 227965 & 589 & 0.259 & 72 & 228037 & 661 & 0.291 \\
\hline 010110 & 75880 & 76109 & 229 & 0.301 & 90 & 76198 & 318 & 0.420 \\
\hline 010101 & 133513 & 133177 & -336 & -0.252 & 829 & 134006 & 492 & 0.369 \\
\hline 010011 & 148160 & 148340 & 180 & 0.121 & 372 & 148712 & 552 & 0.372 \\
\hline 001110 & 135788 & 136536 & 748 & 0.551 & -213 & 136323 & 535 & 0.394 \\
\hline 001101 & 193681 & 193604 & -76 & -0.039 & 526 & 194130 & 450 & 0.232 \\
\hline 001011 & 208255 & 208767 & 512 & 0.246 & 69 & 208837 & 582 & 0.279 \\
\hline 000111 & 114376 & 113979 & -397 & -0.347 & 826 & 114805 & 429 & 0.375 \\
\hline \multicolumn{9}{|c|}{ coalitions of 4 countries } \\
\hline 111100 & 233398 & 234720 & 1322 & 0.566 & -493 & 234227 & 829 & 0.355 \\
\hline 111010 & 247830 & 249883 & 2053 & 0.828 & -949 & 248933 & 1104 & 0.445 \\
\hline 111001 & 306113 & 306951 & 838 & 0.274 & -210 & 306741 & 628 & 0.205 \\
\hline 110110 & 154332 & 155095 & 763 & 0.494 & -192 & 154902 & 571 & 0.370 \\
\hline 110101 & 212255 & 212163 & -92 & -0.043 & 546 & 212710 & 454 & 0.214 \\
\hline 110011 & 226825 & 227326 & 501 & 0.221 & 90 & 227416 & 591 & 0.261 \\
\hline 101110 & 214285 & 215522 & 1237 & 0.577 & -495 & 215027 & 741 & 0.346 \\
\hline 101101 & 272543 & 272590 & 48 & 0.018 & 244 & 272834 & 292 & 0.107 \\
\hline 101011 & 286996 & 287753 & 757 & 0.264 & -213 & 287540 & 544 & 0.190 \\
\hline 100111 & 193119 & 192965 & -154 & -0.080 & 544 & 193509 & 390 & 0.202 \\
\hline 011110 & 178761 & 179758 & 998 & 0.558 & -334 & 179425 & 664 & 0.372 \\
\hline 011101 & 236817 & 236827 & 10 & 0.004 & 405 & 237232 & 415 & 0.175 \\
\hline 011011 & 251338 & 251990 & 652 & 0.259 & -51 & 251938 & 600 & 0.239 \\
\hline 010111 & 157457 & 157202 & -255 & -0.162 & 706 & 157907 & 451 & 0.286 \\
\hline 001111 & 217685 & 217629 & -57 & -0.026 & 403 & 218032 & 346 & 0.159 \\
\hline \multicolumn{9}{|c|}{ coalitions of 5 countries } \\
\hline 111110 & 257284 & 258744 & 1461 & 0.568 & -616 & 258129 & 845 & 0.328 \\
\hline 111101 & 315738 & 315813 & 75 & 0.024 & 123 & 315936 & 198 & 0.063 \\
\hline 111011 & 330123 & 330976 & 853 & 0.258 & -333 & 330642 & 519 & 0.157 \\
\hline 110111 & 236267 & 236188 & -79 & -0.033 & 423 & 236611 & 344 & 0.146 \\
\hline 101111 & 296612 & 296615 & 3 & 0.001 & 121 & 296736 & 124 & 0.042 \\
\hline 011111 & 260851 & 260851 & 1 & 0.000 & 282 & 261134 & 283 & 0.108 \\
\hline \multicolumn{9}{|c|}{ coalitions of 6 countries } \\
\hline 111111 & 339837 & 339837 & 0 & 0.000 & 0 & 339837 & 0 & 0.000 \\
\hline
\end{tabular}




\subsection{Checking the core property of the generalized GTT transfers}

In column six of Table 2, generalized GTT transfers $\widetilde{\Psi}_{S}$ are reported as computed from formula (29) with shares adjusted as in (30). Column seven $\left(W_{S}^{*}+\widetilde{\Psi}_{S}\right)$ contains the value of total consumption available to the coalitions after these transfers have taken place. The last two columns show the differences $W_{S}^{*}+\widetilde{\Psi}_{S}-W_{S}^{S}$ in absolute amounts and in percentages. As the transfers $\widetilde{\Psi}_{i}$ should balance, we verify that $\sum_{N} \widetilde{\Psi}_{i}=0$ in the last line of Table 2 . The shares of the regions in the surplus of cooperation $\widetilde{\pi}_{i}$ are as follows: USA: $19.8 \%, J P N$ : 10.8\%, EU: 28.0\%, CHN: 3.0\%, FSU: $6.1 \%$ and ROW:32.3\%. Hence, EU and ROW seize each about $30 \%$ of the surplus of cooperation. Recall that these shares reflect the different regions' share in total World discounted marginal climate change damages. These weights resemble closely the distribution of GDP in the reference year 1990 (see Table 6 in the Appendix).

Table 2 shows that the industrialized regions ( $U S A, J P N, E U$ and $F S U$ ) pay transfers to the developing regions $C H N$ and $R O W$. In particular, the transfer scheme (29) compensates $C H N$ and $R O W$ such that they are better off under the Pareto efficient allocation with transfers than if there were no cooperation at all. Hence, both regions have no incentive to deviate individually anymore. Eventually, all regions are individually better off under the transfer scheme compared to the non-cooperative open-loop Nash equilibrium since the difference $W_{S}^{*}+\widetilde{\Psi}_{S}-W_{S}^{S}$ is positive for all regions. Hence, the transfer solution satisfies individual rationality.

It can also be seen from Table 2 that the coalitional rationality constraints are met for all possible subcoalitions $S \subset N$ with $\# S \geq 2$. In particular the twelve coalitions for which coalitional rationality was violated in the Pareto efficient allocation without transfers receive sufficient compensation. Hence, the allocation with GTT transfers is a core allocation for the emission abatement game associated to the CWS model ${ }^{16}$.

\subsection{Coalitional aspects of some post-Kyoto policies}

In addition to providing a numerical proof of the core property of the efficient scenario with GTT transfers, Table 2 is also an instrument for evaluating policies that single countries, or coalitions of countries, might consider adopting. As examples, let us single out the following three such policies, which happen to be of particular interest in the current context:

\footnotetext{
${ }^{16}$ We have run numerous simulations for different sets of parameters like discount rates, climate change damage parameters, free riding behavior in region $R O W$ etc. Details on this sensitivity analysis can be obtained from the authors. For none of these sensitivity analysis we found a violation of the core property of the transfer scheme. Of course, this is not a general proof of the core property for nonlinear damage functions but still it indicates that the result is robust.
} 
1. The "Kyoto Annex B countries" policy, where coalition $S$ corresponds to the line keyed as $\{111010\}$ (see coalitions of 4 countries, line two). The table reveals that for this coalition,

$$
W_{S}^{S}=247.830>\sum_{i \in S} W_{\{i\}}^{\{i\}}=247.787
$$

that is, the aggregate payoff for the Annex B coalition in terms of discounted aggregate consumption is higher than if each of its members chooses the NASH scenario. Thus, although this coalition does not achieve optimality at the World level, its formation is preferable for all its members to isolated domestic actions (NASH). It is thus collectively rational for the Annex B countries to act on their own, as specified by the Kyoto Protocol, in spite of the fact that the rest of the World sticks to an individualistic, non cooperative policy.

2. The "George W. Bush" policy, where the coalition $S$ corresponds to the line keyed as $\{100000\}$. Table 2 reveals that for this coalition,

$$
W_{U S A}^{U S A}=78.353<W_{U S A}^{*}+\Psi_{U S A}=78.704<W_{U S A}^{*}=78.986
$$

that is, sticking to a domestic optimization policy only is for the $U S A$ less beneficial than the World optimal policy, both with and without transfers.

3. The "Annex B without USA" policy, where the coalition $S$ corresponds to the line keyed as $\{011010\}$ (coalitions of 3 countries, line 12). For this coalition,

$$
W_{S}^{S}=169.448>\sum_{i \in S} W_{\{i\}}^{\{i\}}=169.434
$$

meaning that, in spite of the Bush policy of staying out of the Protocol, the Annex B group would benefit from the partial cooperation achieved among the non-USA countries. This comes as a support of the policy initiated by the EU in the Spring of 2001. Our simulations show also that the USA gains only very little from leaving the Kyoto coalition. By renouncing the Kyoto coalition (and free riding on the effort of the remaining Kyoto members), the $U S A$ 's pay off would be $W_{U S A}^{S}=78.367$ for $\mathrm{S}=\{011010\}$, which is only slightly more than what it would achieve if it would stick to its Kyoto commitment $\left(W_{U S A}^{S}=78.366 \text { for } \mathrm{S}=\{111010\}\right)^{17}$.

\subsection{The transfers and time}

Figure 9 shows the differences in World consumption levels, at each point in time $t$, between the non-cooperative Nash equilibrium and the Pareto efficient scenarios: $\sum_{j \in N}\left[Z_{j, t}^{*}-\right.$

\footnotetext{
${ }^{17}$ Moreover, in Eyckmans (2001), it is shown that the deviation of USA from the Kyoto Protocol is not credible in the farsighted sense as defined by Chwe (1994). The initial deviation of the USA would trigger of subsequent deviations by, among others, the FSU, making the United States in the end worse off than under the original Kyoto Protocol. Further coalitional analysis of the CWS model is offered in Eyckmans and Finus (2003).
} 
Figure 9: EFF-NASH difference in World consumption (billion 1990US\$)

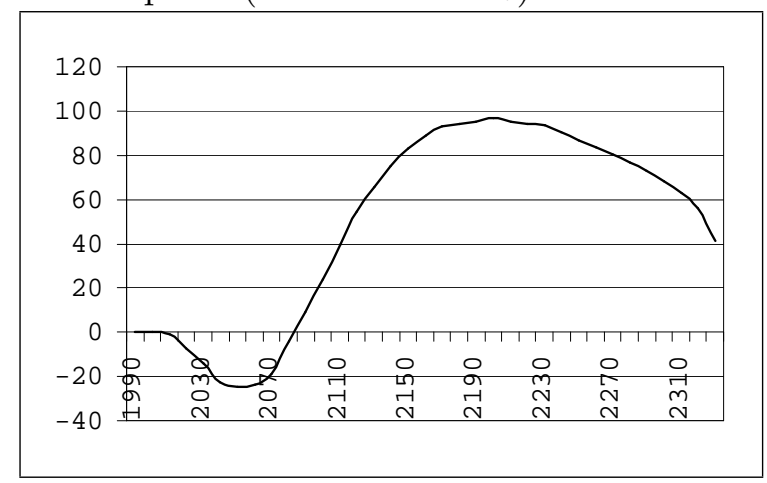

$\left.Z_{j, t}^{N E}\right]\left[1+\rho_{j}\right]^{-t}$. All values are discounted back to 1990. Hence, Figure 9 depicts the evolution over time of the value of cooperation over noncooperation. Typically, up to the year 2080, the NASH scenario dominates the efficient scenario EFF. After 2090 however, the order of dominance is reversed. In total, the sum over time of all differences (the surface under the curve) is positive as the gains from cooperation in the far future more than compensate for the initial losses. This means that we are in a situation as in Assumption 3 in GTT. Obviously, the countries cannot borrow against future gains in order to compensate for early losses. We should therefore design a transfer scheme in such a way that the regions most affected initially be compensated partially by the less affected regions. An attempt to design such a transfer scheme with transfers evolving over time is reported, in theoretical terms, in Germain, Toint, Tulkens and De Zeeuw (1998, forthcoming 2003) where the appropriate tool for that purpose is found to be closed loop Nash equilibria replacing open-loop ones. However, computational complexity of this scheme requires further research before it can be applied to the CWS model.

\section{Conclusion}

As briefly alluded to in the introduction, there are game theoretic approaches to the climate change problem that conclude with scepticism about the chance of ever reaching World wide cooperation in this area. The present paper concludes with the opposite view. This difference in results stems from using different branches of game theory. One of these branches dealing with stability of coalitions, inspires the former approach; another branch - cooperative (or coalitional) game theory, provides the foundation for the present paper. Evaluating the respective degrees of validity of either approaches must be left to a pure game theoretical paper, still to be written. 
In the meantime, this paper contributes to that debate in a way whose generality is wider than most papers defending the sceptic thesis. Here, conditions for World cooperation are obtained in the framework of a dynamic, multi-regional integrated assessment model including stock externalities and numerical verification. The progress brought about by these features over the one-equation models used in the other approach does arguably give more credibility to the cooperative approach advocated here.

\section{$7 \quad$ References}

Barrett, S., 1994, Self-enforcing international environmental agreements, Oxford Economic Papers 46, 878-894

Carraro, C. and D. Siniscalco, 1993, Strategies for the international protection of the environment, Journal of Public Economics 52, 309-328

Chander, P. and H. Tulkens, 1995, A core-theoretic solution for the design of cooperative agreements on transfrontier pollution, International Tax and Public Finance 2, 279 293

Chander, P. and H. Tulkens, 1997, The core of an economy with multilateral environmental externalities, International Journal of Game Theory 26, 379-401

Chander, P., H. Tulkens, J.-P. van Ypersele and S. Willems, 2002, The Kyoto Protocol, an economic and game theoretic interpretation, chapter 6 in: P. Dasgupta, B. Kriström and K.G. Löfgren, eds., Environmental Economics: Theoretical and Empirical Inquiries, Festschrift in honour of Karl Göran Mäler (Edward Elgar, Celtenham UK Brookfield US) 98-117

Chwe, M. S.-Y., 1994, Farsighted coalitional stability, Journal of Economic Theory 63, 299325

Eyckmans, J., 1997, Nash implementation of a proportional solution to international pollution control problems, Journal of Environmental Economics and Management 33, 314330

Eyckmans, J., 2001, On the farsighted stability of the Kyoto Protocol, ETE Working Paper 2001-03 (K.U.Leuven-CES-ETE) and CLIMNEG Working Paper 40 (UcL, Louvain-la-Neuve)

Eyckmans, J., S. Proost and E. Schokkaert, 1993, Equity and efficiency in greenhouse negotiations, Kyklos 46, 363-397 
Eyckmans, J. and C. Bertrand, C., 2000, Integrated assessment of carbon and sulphur emissions, simulations with the CLIMNEG model, CLIMNEG Working Paper 32 (UcL, Louvain-la-Neuve)

Eyckmans, J. and M. Finus, 2003, Coalition Formation in a Global Warming Game: How the Design of Protocols Affects the Success of Environmental Treaty-Making, mimeo

Germain, M., P.L. Toint and H. Tulkens, 1997, Financial transfers to ensure international optimality in stock pollutant abatement, in: F. Duchin, S. Faucheux, J. Gaudy I. and Nicolaï, eds., Sustainability and firms: technological change and changing regulatory environment (Edward Elgar, Celtenham UK - Brookfield US)

Germain, M., P.L. Toint, H. Tulkens and A. De Zeeuw, forthcoming, Transfers to sustain core-theoretic cooperation in international stock pollutant control, Journal of Economic Dynamics and Control

Hackl, F. and G.J. Pruckner, 2002, How global is the solution to global warming? Economic Modelling 20, 93-117

Hoel, M., 1992, International environmental conventions: the case of uniform reductions of emissions, Environmental and Resource Economics 2, 141-159

Kaitala, V., M. Pohjola and O. Tahvonen, 1992, Transboundary air pollution and soil acidification: A dynamic analysis of an acid rain game between Finland and the USSR, Environmental and Resource Economics 2, 161-181

Mäler, K.G., 1989, The acid rain game, in: H. Folmer, and E. Van Ierland, eds., Valuation Methods and Policy Making in Environmental Economics (Elsevier Science Publishers, Amsterdam) 231-252

Nordhaus, W.D. and Z. Yang, 1996, A regional dynamic general-equilibrium model of alternative climate-change strategies, American Economic Review 86, 741-765

Samuelson, P.A., 1954, The pure theory of public expenditure, Review of Economics and Statistics 36, 387-389

Tulkens, H., 1979, An economic model of international negotiations relating to transfrontier pollution, in: K. Krippendorff, ed., Communication and Control in Society (Gordon and Breach Science Publishers, New York-London) 199-212

Tulkens, H., 1998, Cooperation versus free-riding in international environmental affairs: two approaches, in: N. Hanley, and H. Folmer, eds., Game Theory and the Environment (Edward Elgar, Celtenham UK - Brookfield US) 30-44

van der Ploeg, F., and De Zeeuw, A.J., 1992, International aspects of pollution control, Environmental and Resource Economics 2, 117-139 
Yang, Z., 1998, Incentive compatible coalitions in international agreements on $\mathrm{CO}_{2}$ emission controls, mimeo MIT (paper presented at NBER workshop in Boston, August 1998)

\section{Appendix}

Equation listing of the CWS model

$$
\begin{aligned}
U_{i}\left(Z_{i, t}\right) & =Z_{i, t} \\
Y_{i, t} & =Z_{i, t}+I_{i, t}+C_{i}\left(\mu_{i, t}\right)+D_{i}\left(\Delta T_{t}\right) \\
Y_{i, t} & =A_{i, t} K_{i, t}^{\gamma} L_{i, t}^{1-\gamma} \\
C_{i}\left(\mu_{i, t}\right) & =Y_{i, t} b_{i, 1} \mu_{i, t}^{b_{i, 2}} \\
D_{i}\left(\Delta T_{t}\right) & =Y_{i, t} \theta_{i, 1} \Delta T_{t}^{\theta_{i, 2}} \\
K_{i, t+1} & =\left[1-\delta_{K}\right] K_{i, t}+I_{i, t} \quad K_{i, 0} \text { given } \\
E_{i, t} & =\sigma_{i, t}\left[1-\mu_{i, t}\right] Y_{i, t} \\
M_{t+1} & =\left[1-\delta_{M}\right] M_{t}+\beta \sum_{i \in N} E_{i, t} \quad M_{0} \text { given } \\
F_{t} & =\frac{4.1 \ln \left(M_{t} / M_{0}\right)}{\ln (2)}+F_{t}^{x} \\
T_{t}^{o} & =T_{t-1}^{o}+\tau_{3}\left[\Delta T_{t-1}-T_{t-1}^{o}\right] \\
\Delta T_{t} & =\Delta T_{t-1}+\tau_{1}\left[F_{t}-\lambda \Delta T_{t-1}\right]-\tau_{2}\left[\Delta T_{t-1}-T_{t-1}^{o}\right]
\end{aligned}
$$


Table 3: List of variables

\begin{tabular}{ll}
\hline$Y_{i, t}$ & production (billion 1990 US\$) \\
$A_{i, t}$ & productivity \\
$Z_{i, t}$ & consumption (billion 1990 US\$) \\
$I_{i, t}$ & investment (billion 1990 US\$) \\
$K_{i, t}$ & capital stock (billion 1990 US $\$$ ) \\
$L_{i, t}$ & population (million people) \\
$C_{i, t}$ & cost of abatement (billion 1990 US $\$$ ) \\
$D_{i, t}$ & damage from climate change (billion 1990 US\$) \\
$E_{i, t}$ & carbon emissions (billion tons of C) \\
$\sigma_{i, t}$ & emission-output rate \\
$\mu_{i, t}$ & emission abatement \\
$M_{t}$ & atmospheric carbon concentration $($ billion tons of C) \\
$F_{t}$ & radiative forcing (Watt per $\left.\mathrm{m}^{2}\right)$ \\
$\Delta T_{t}$ & temperature increase atmosphere $\left({ }^{\circ} \mathrm{C}\right)$ \\
$T_{t}^{o}$ & temperature increase deep ocean $\left({ }^{\circ} \mathrm{C}\right)$ \\
\hline
\end{tabular}


Table 4: Global parameter values

\begin{tabular}{llr}
\hline$\delta_{K}$ & capital depreciation rate & 0.10 \\
$\gamma$ & capital productivity parameter & 0.25 \\
$\beta$ & airborne fraction of carbon emissions & 0.64 \\
$\delta_{M}$ & atmospheric carbon removal rate & 0.0833 \\
$\tau_{1}$ & parameter temperature relationship & 0.226 \\
$\tau_{2}$ & parameter temperature relationship & 0.44 \\
$\tau_{3}$ & parameter temperature relationship & 0.02 \\
$\lambda$ & parameter temperature relationship & 1.41 \\
$M_{0}$ & initial carbon concentration & 590 \\
$\Delta T_{0}$ & initial temperature change atmosphere & 0.50 \\
$T_{0}^{o}$ & initial temperature change deep ocean & 0.10 \\
\hline
\end{tabular}


Table 5: Regional parameter values

\begin{tabular}{lccccc}
\hline & $\theta_{i, 1}$ & $\theta_{i, 2}$ & $b_{i, 1}$ & $b_{i, 2}$ & $\rho_{i}$ \\
\hline USA & 0.01102 & 2.0 & 0.07 & 2.887 & 0.015 \\
JPN & 0.01174 & 2.0 & 0.05 & 2.887 & 0.015 \\
EU & 0.01174 & 2.0 & 0.05 & 2.887 & 0.015 \\
CHN & 0.01523 & 2.0 & 0.15 & 2.887 & 0.030 \\
FSU & 0.00857 & 2.0 & 0.15 & 2.887 & 0.015 \\
ROW & 0.02093 & 2.0 & 0.10 & 2.887 & 0.030 \\
\hline
\end{tabular}


Table 6: 1990 reference year variables

\begin{tabular}{lcrrrrrrr}
\hline & $Y_{i}^{0}$ & \multicolumn{1}{c}{$(\%)$} & \multicolumn{1}{c}{$K_{i}^{0}$} & \multicolumn{1}{c}{$(\%)$} & \multicolumn{1}{c}{$L_{i}^{0}$} & \multicolumn{1}{c}{$(\%)$} & $E_{i}^{0}$ & $(\%)$ \\
\hline USA & $5,464.796$ & 25.9 & $14,262.510$ & 26.3 & 250.372 & 4.8 & 1.360 & 20.5 \\
JPN & $2,932.055$ & 13.9 & $8,442.250$ & 15.6 & 123.537 & 2.4 & 0.292 & 10.9 \\
EU & $6,828.042$ & 32.4 & $18,435.710$ & 34.0 & 366.497 & 7.0 & 0.872 & 28.9 \\
CHN & 370.024 & 1.8 & $1,025.790$ & 1.9 & $1,133.683$ & 21.5 & 0.669 & 3.0 \\
FSU & 855.207 & 4.1 & $2,281.900$ & 4.2 & 289.324 & 5.5 & 1.066 & 6.8 \\
ROW & $4,628.621$ & 22.0 & $9,842.220$ & 18.1 & $3,102.689$ & 58.9 & 1.700 & 29.9 \\
\hline World & $21,078.750$ & 100.0 & $54,290.380$ & 100.0 & $5,266.100$ & 100.0 & 5.959 & 100.0 \\
\hline & billion & $(\%)$ & billion & $(\%)$ & million & $(\%)$ & gigaton & $(\%)$ \\
& US $\$$ 1990 & & US $\$ 1990$ & & people & & carbon GtC &
\end{tabular}

